\title{
Role of Multidetector Computed Tomography in Gastric Cancers
}

\author{
Eisha Ramadan Mohamed, Alsiagy A. Abdel-Aziz, Eman Mohammed Mohammed Lofty* \\ Departments of Radiodiagnosis, Faculty of Medicine - Alazhar University \\ *Correspondence author: Eman Mohammed Mohammed Lofty, Mobile: (+20) 01006287509, \\ E-mail: emanlotfy1000@gmail.com
}

\begin{abstract}
Background: Gastric cancer is the third most common cause of cancer-related death in the world. Currently, multislice computed tomography (MSCT) is a valuable tool for detection, staging, surveillance, and post-treatment evaluation of gastric neoplasm. Objective: The aim of this work is to clarify the role of multidetector CT in diagnosis and preoperative staging of gastric carcinoma. Patients and methods: The present study was conducted between October 2017 and June 2019 on 60 patients (26 males and 34 females) with age ranged from 28 to 73 years with mean age of 53 years. The patients were complaining of symptoms of gastric cancer or as a follow-up study for gastric cancer, referred to the CT Unit in Tanta University Hospital, Tanta Oncology Center and Health Insurance Hospital from the Oncology Department. Results: In our study we found that there was a significant relationship between pathological and CT staging by using MPR. CT with MPR was specific and accurate in diagnosis of all stages of gastric cancer with specificity ranged between (95-100\%) and accuracy ranged between (94.5-97.5\%). However, it showed lowest sensitivity in diagnosis of stage 1 of gastric cancer. On the other hand, it showed highest sensitivity (97.5\%) in diagnosis of stage IV. Conclusion: MSCT is a valuable tool for detection, staging, surveillance, and post-treatment evaluation of gastric neoplasm.
\end{abstract}

Keywords: Multi-detector Computed Tomography, Gastric Cancers.

\section{INTRODUCTION}

Although diagnostic advancements in performing endoscopy and double-contrast barium study currently allows the detection of small lesions early in the course of the disease, the depth of tumor invasion and the presence or absence of metastases could not be determined with either modality. In a routine clinical setting, patients with esophageal and gastric carcinoma most commonly undergo diagnosis and staging with computed tomography ${ }^{(\mathbf{1})}$.

Multidetector-row computed tomography, particularly those apparatuses with 16 or more channels, offers rapid acquisition of submillimetric sections, isotropic multiplanar reconstruction and post processing options such as virtual endoscopy, which increases the method accuracy in the local staging (2). Additionally, computed tomography can evaluate lymph nodes and other organs ${ }^{(3)}$.

MSCT can provide valuable additional information and improve the detection and staging of both early and advanced gastric neoplasm. MSCT offers high diagnostic accuracy in tumor detection and staging of lymph node metastasis, and high reliable information as regards secondary tumors. MSCT is a valuable tool for monitoring response to treatment and post-treatment evaluation of gastric neoplasm ${ }^{(4)}$.

The most recent international consensus corroborated the necessity of preoperative TNM staging and pointed out multidetector-row computed tomography as the best staging method, which demonstrated similar or superior accuracy as compared with endoscopic ${ }^{\bullet}$ ultrasonography for T-staging and a clear advantage in relation to other methods for $\mathrm{N}$ - and $\mathrm{M}$-staging ${ }^{(5)}$.

The role of multidetector computed tomography (MDCT) is to differentiate between benign and malignant ${ }^{\bullet}$ gastric neoplasm and determine the stage and gastric spread of gastric carcinoma, which is vital in choosing between palliative or radical surgical treatment. In addition, MDCT is used to monitor response to treatment. Furthermore, it was shown to be a very important prognostic factor in patients with gastric cancer by evaluation and estimation of tumor invasion depth after multiplaner reconstruction (MPR) ${ }^{(4)}$.

\section{AIM OF THE WORK}

The aim of this work is to clarify the role of multidetector CT in diagnosis and preoperative staging of gastric carcinoma.

\section{PATIENTS AND METHODS}

The present study was conducted between October 2017 and June 2019 on 60 patients (26 males and 34 females) with age ranged from 28 to 73 years with mean age of 53 years. The patients were complaining of symptoms of gastric cancer or as a follow-up study for gastric cancer, referred to the CT Unit in Tanta University Hospital, Tanta Oncology Center and Health Insurance Hospital from the Oncology Department.

\section{Written informed consent:}

An approval of the study was obtained from Al- Azhar University academic and ethical committee. Every patient signed an informed written consent for acceptance of the operation.

\section{All patients were subjected to the following:}

Detailed history taking including: history of smoking, family history of gastric carcinoma and past history of previous gastric surgery or radiation exposure (Data were taken from patients' files).

Clinical examination and laboratory investigations. 
- Multidetector computed tomography of the abdomen and pelvis.

\section{Technique:}

CT examinations were performed using a multidetector 128 rows helical CT scanner (Siemens). Before CT examination, each patient was prepared by overnight fasting to empty the stomach. Butyl scopolamine (Buscopan) (10 mg) was administered intravenously in all patients to reduce bowel peristalsis. Patients were advised to ingest two packs of effervescent granules with minimal water to obtain gastric distension. All patients ingested oral contrast media in the form (300-600 $\mathrm{ml}$ of tap water to distend the stomach). This allowed good visualization of the enhancing gastric wall and avoids overshooting artifacts due to intraluminal air obscuring the normal gastric wall pattern. After a scout projection, CT scanning was performed from the diaphragmatic dome to the level of the symphysis pubis during a single breath-hold of 7-10 seconds.

Unenhanced CT scanning was done in supine position. Scanning CT parameters were $16 \times 1.25 \mathrm{~mm}$ beam collimation, $1.2 \mathrm{~mm}$ slice thickness, $2.5 \mathrm{~mm}$ reconstruction interval, a pitch of $\mathrm{mm}$, gantry rotation time $0.5 \mathrm{Sec}$, table feed of $7.5 \mathrm{~mm} /$ rotation, field of view $30-35 \mathrm{~cm}, 120 \mathrm{kVp}$ and $80 \mathrm{~mA}$. Each patient then received an intravenous dose of $1-2 \mathrm{ml} / \mathrm{kg}$ of non-ionic water soluble contrast material (Omnipaque) which was administered by power injector at a flow rate of $3 \mathrm{ml} / \mathrm{sec}$ through an 18-F plastic catheter placed in antecubital vein using a dual-phase technique. For post-contrast CT, the tube current was increased to $210 \mathrm{~mA}$ to improve the spatial resolution. MDCT scanning for the acquisition of the first sequence during the arterial phase was started 25-30 s after initiating injection of contrast medium, with the second sequence started 55-60 second later during the portal phase using bolus tracking software for time adjustment. This protocol allows optimal visualization of both gastric arteries and veins. Arterial phase accentuated the difference in enhancement between normal gastric wall layers and tumor and also displayed the gastric arterial anatomy, while, the portal phase identified the depth of tumor invasion through the deeper portion of the stomach wall. The CT dataset of each patient was transferred to an image processing workstation. Sagittal and coronal images of $0.6 \mathrm{~mm}$ thickness were obtained for all patients.

All MDCT images were carefully reviewed and irregular wall thickness (> $8 \mathrm{~mm}$ ) was considered abnormal. Any gastric mass with or without wall thickness was also assessed for location, size, enhancement pattern and extragastric spread. If the mucosal layer was intact over a mass, a submucosal tumor was considered. If the mucosal layer was unevenly thickened and showed abnormal enhancement, a mucosal lesion or gastric cancer was diagnosed. Ulceration was considered malignant when focal interruption of mucosa with adjacent nodularity or thickening was found. If only focal interruption of the mucosal layer was found, a benign gastric ulcer was considered. Scans were also reviewed for associated lymph node enlargement, distant metastasis and ascites.

Patients diagnosed as having gastric carcinoma by MDCT were staged according to the American Joint Committee of Cancer (AJCC) staging system as follows:

In T1 and T2 lesions, invasion is limited to the gastric wall. In T3 lesions, the serosal contour becomes blurred, and strand-like areas of increased attenuation were extending into the perigastric fat, and in T4 lesions, tumor spread frequently occurs via ligamentous and peritoneal reflections to adjacent organs. Positive nodes were identified on the basis of size $(>10 \mathrm{~mm}$ along the short axis), shape (round shape with or without central necrosis), and enhancement pattern (marked or heterogeneous enhancement). Regarding N classification; N1, means metastasis in one to six regional lymph nodes; $\mathrm{N} 2$, means metastasis in seven to 15 lymph nodes; and N3, metastasis in more than 15 lymph nodes. Regarding M classification; M0, means lesions not associated with distant metastases while M1, means lesions associated with distant metastases.

TNM classification system for staging gastric carcinoma:

- Stage 0: Tis, N0, M0.

- Stage IA: T1, N0 or N1, M0.

- Stage IB: T1, N2, M0 or T2, N0, M0.

- Stage II: T1, N2, M0 or T2, N1, M0 or T2, N0, M0.

- Stage IIIA: T2, N2, M0 or T3, N1, M0 or T4, N0, M0.

- Stage IIIB: T3, N2, M0.

- Stage IV: T1-3, N3, M0 or T4, N1-3, M0, or any T, any $\mathrm{N}, \mathrm{M} 1$.

MDCT findings were compared with the histopathologic results obtained from endoscopic biopsies and/or intraoperative surgery specimen.

\section{Statistics}

Statistical presentation and analysis of the present study was conducted, using chi-square test by SPSS, V.16 for comparison between two groups as regard qualitative data. Sensitivity, specificity, PPV (positive predictive value), NPV (negative predictive value), and accuracy were also calculated. $\mathrm{P}<0.05$ was considered significant.

\section{RESULTS}

Sixty patients were evaluated in this study. The final diagnosis was obtained depended on further endoscopic biopsy and operative data.

Table (1): The distribution of age frequency of the studied patients $(\mathrm{n}=60)$.

\begin{tabular}{||c|c|c|}
\hline \multirow{2}{*}{ Age in years } & \multicolumn{2}{|c|}{ Patients } \\
\cline { 2 - 3 } & No. & \% \\
\hline $\mathbf{2 0 - 3 0}$ & 1 & 1.7 \\
\hline$>\mathbf{3 0 - 4 0}$ & 10 & 16.7 \\
\hline $\mathbf{4 0 - 5 0}$ & 6 & 10 \\
\hline$>\mathbf{5 0 - 6 0}$ & 20 & 33.3 \\
\hline$>\mathbf{6 0 - 7 0}$ & 20 & 33.3 \\
\hline$>\mathbf{7 0 - 8 0}$ & 3 & 5 \\
\hline Total & 60 & 100 \\
\hline
\end{tabular}

The most frequently involved age group was the group > 50- 70 years. 
Table (2): the distribution of Age and Sex frequency of the studied patients (60).

\begin{tabular}{|c|c|c|c|c|c|c|}
\hline \multirow{3}{*}{ Age in years } & \multicolumn{4}{|c|}{ Sex } & \multirow{2}{*}{\multicolumn{2}{|c|}{ Total }} \\
\hline & \multicolumn{2}{|c|}{ Male } & \multicolumn{2}{|c|}{ Female } & & \\
\hline & No. & $\%$ & No. & $\%$ & No. & $\%$ \\
\hline $20-30$ & 1 & 1.7 & 0 & 0 & 1 & 1.7 \\
\hline$>30-40$ & 1 & 1.7 & 9 & 15 & 10 & 16.7 \\
\hline$>40-50$ & 3 & 5 & 3 & 5 & 6 & 10 \\
\hline$>50-60$ & 16 & 26.7 & 4 & 6.7 & 20 & 33.3 \\
\hline$>60-70$ & 2 & 3.3 & 18 & 30 & 20 & 33.3 \\
\hline$>70-80$ & 3 & 5 & 0 & 0 & 3 & 5 \\
\hline Total & 26 & 43.3 & 34 & 56.7 & 60 & 100 \\
\hline
\end{tabular}

By analyzing table 2 among patients with gastric carcinoma, we found that gastric cancers are more common in females than males.

Table (3): Clinical presentations of the gastric cancers patients (60)

\begin{tabular}{|c|c|c|}
\hline \multirow[b]{2}{*}{ Clinical Presentation } & \multicolumn{2}{|c|}{ Carcinoma Patients ( $N=60)$} \\
\hline & No & $\%$ \\
\hline - Abdominal Pain & 30 & 50 \\
\hline - Progressive loss of weight & 30 & 50 \\
\hline - Vomiting Colic & 25 & 41.6 \\
\hline - Epigastric pain & 7 & 11.6 \\
\hline - Dyspepsia & 6 & 10 \\
\hline - Hematemesis & 4 & 6.7 \\
\hline - Fever & 3 & 5 \\
\hline - Loss of Appetite & 2 & 3.3 \\
\hline - Dysphagia & $\begin{array}{l}2 \\
1\end{array}$ & 3.5 \\
\hline - Nausea & 1 & 1.7 \\
\hline - Black Stool & 1 & 1.7 \\
\hline
\end{tabular}

From the previous table we found that the most frequently encountered clinical presentations among gastric carcinoma patients were abdominal pain and progressive weight loss.

Table (4): Final diagnoses and methods used in the studied 60 patients.

\begin{tabular}{|c|c|c|}
\hline $\begin{array}{c}\text { Methods used for final diagnosis } \\
\text { of UB carcinoma }\end{array}$ & No. & \% \\
\hline Gastroscopic biopsy & 57 & $95 \%$ \\
\hline Postoperative histopathology & 3 & $5 \%$ \\
\hline Total & 60 & $100 \%$ \\
\hline
\end{tabular}

All the studied 60 patient had malignant stomach lesions and were finally diagnosed as gastric carcinomas, the final diagnoses were based on the findings of gastroscopic biopsy in 57 (95\%) patients, and postoperative histopathological examination in $3(5 \%)$ patients.

Table (5): The distribution of pathological types of gastric cancer of the studied 60 patients.

\begin{tabular}{|c|c|c|}
\hline Types of gastric cancer & No. & \% \\
\hline Poorly differentiated adenocarcinoma & 20 & $33.3 \%$ \\
\hline High grade invasive adenocarcinoma & 15 & $25 \%$ \\
\hline Round cell carcinoma & 10 & $16.7 \%$ \\
\hline Signet ring carcinoma & 10 & $16.7 \%$ \\
\hline Toderately differentiated adenocarcinoma & 5 & $8.3 \%$ \\
\hline Total & 60 & $100 \%$ \\
\hline
\end{tabular}

Table 5 detected that the undifferentiated adenocarcinoma is the most common gastric cancer. 
Table (6): Multislice CT imaging shape of gastric cancer in the studied 60 patients.

\begin{tabular}{||c|c|c|c|c|c|c||}
\hline $\begin{array}{l}\text { Shape of gastric } \\
\text { cancer by MDCT }\end{array}$ & \multicolumn{2}{|c|}{ Males } & \multicolumn{2}{c|}{ Females } & \multicolumn{2}{c|}{ Total } \\
\cline { 2 - 7 } \\
\hline $\begin{array}{l}\text { Polypoidal or } \\
\text { fungating mass }\end{array}$ & 11 & $18.3 \%$ & 10 & $16.7 \%$ & 21 & $35 \%$ \\
\hline Ulcerating lesion & 2 & $3.3 \%$ & 2 & $3.3 \%$ & 4 & 6.7 \\
\hline $\begin{array}{l}\text { Focal wall } \\
\text { thickening }\end{array}$ & 8 & $13.3 \%$ & 7 & $11.7 \%$ & 15 & $25 \%$ \\
\hline $\begin{array}{l}\text { Circumferential } \\
\text { wall thickening }\end{array}$ & 5 & $8.3 \%$ & 15 & $25 \%$ & 20 & $33.3 \%$ \\
\hline Total & 26 & $43.3 \%$ & 34 & $56.7 \%$ & 60 & $100 \%$ \\
\hline
\end{tabular}

All sixty patients were scanned by multislice CT. Gastric mass appeared in 21 cases (Table 6).

Table (7): The relation between pathological and CT staging according to depth of the invasion with MPR.

\begin{tabular}{|l|c|c|c|c|}
\hline \multirow{2}{*}{ CT staging } & \multicolumn{4}{|c|}{ Pathological staging } \\
\cline { 2 - 5 } & PT1(N=4) & PT2(N=6) & PT3(N=18) & PT4(N=32) \\
\hline T1(N=6) & 6 & - & - & - \\
\hline T2(N=8) & 2 & 6 & - & - \\
\hline T3(N=13) & - & 1 & 12 & 31 \\
\hline T4(N=33) & - & - & 2 & \\
\hline $\mathbf{X}^{2}$ & \multicolumn{3}{|c|}{$\mathbf{0 . 0 2 2}$} \\
\hline P value & \multicolumn{5}{|c|}{} \\
\hline
\end{tabular}

PT: pathological T: CT tumor staging *significant value (less than 0.05)

Table 7 showed that most of our patients had stage $\mathrm{T} 4(\mathrm{~N}=33)$ according to $\mathrm{CT}$ staging of gastric carcinoma using MPR and pathological staging. The difference between the CT staging and pathological staging of gastric cancers was significant.

Table (8): The tumor detection rate with MDCT axial cuts and MPR in different stages.

\begin{tabular}{|c|c|c|c|c|}
\hline \multirow{3}{*}{$\begin{array}{l}\text { Cancer } \\
\text { staging by } \\
\text { MDCT }\end{array}$} & \multicolumn{4}{|c|}{ Number of patients detected in different stages } \\
\hline & \multicolumn{2}{|c|}{ MPR } & \multicolumn{2}{|c|}{ Axial cuts } \\
\hline & $\mathbf{N}$ & $\%$ & $\mathbf{N}$ & $\%$ \\
\hline T1 & 6 & $1 \%$ & 4 & $6.7 \%$ \\
\hline T2 & 8 & $13.3 \%$ & 8 & $13.3 \%$ \\
\hline T3 & 13 & $21.7 \%$ & 16 & $26.7 \%$ \\
\hline T4 & 33 & $55 \%$ & 32 & $53.3 \%$ \\
\hline $\mathbf{X}^{2}$ & \multicolumn{4}{|c|}{0.637} \\
\hline P value & \multicolumn{4}{|c|}{0.0115} \\
\hline
\end{tabular}

$\mathrm{N}$ (number of patients)

MPR: multiplanar reformation

Table 8 showed that stage 4 of gastric carcinoma was better recognized by using MPR sequences, while grade 1 and 3 of cancer stomach were better identified by using axial cuts with thin slice MDCT however, the difference between the two sequences was not significant.

Table (9): Sensitivity, specificity, PPV, NPV and accuracy of CT in detection of tumor in different stages with MPR.

\begin{tabular}{|l|c|c|c|c|c||}
\hline \hline $\begin{array}{c}\text { CT staging } \\
\text { with MPR }\end{array}$ & sensitivity & Specificity & PPV & NPV & Accuracy \\
\hline T1 (n=6) & 50 & 97 & 66 & 94.5 & 92.5 \\
\hline T2(n=8) & 60 & 97 & 75 & 94 & 92.5 \\
\hline T3(n=13) & 81 & 93 & 82 & 93 & 90 \\
\hline T4(n=33) & 90 & 95 & 95 & 90.4 & 92.5 \\
\hline
\end{tabular}

Table 9 showed that CT was specific and accurate in diagnosis of all stages of gastric carcinoma with specificity ranged between (93\%-97\%) and accuracy ranged between (90\%-92.5\%). However, it showed lowest sensitivity (50\%) in the diagnosis of stage I of gastric cancer. On the other hand it showed highest sensitivity (90\%) in diagnosis of stage 4 . 
Table (10): Sensitivity, specificity, PPV, NPV and accuracy of CT in detection of tumor in different stages with thin slice CT.

\begin{tabular}{|c|c|c|c|c|c|}
\hline $\begin{array}{c}\text { CT staging by } \\
\text { MDCT }\end{array}$ & sensitivity & Specificity & PPV & NPV & Accuracy \\
\hline T1 (n=4) & 75 & 100 & 97.2 & 100 & 94.5 \\
\hline T2(n=8) & 80 & 100 & 97.2 & 100 & 95 \\
\hline T3(n=16) & 91 & 100 & 96.6 & 100 & 96.5 \\
\hline T4(n=32) & 100 & 95.2 & 100 & 95.2 & 97.5 \\
\hline
\end{tabular}

Table 10 showed that CT with MPR was specific and accurate in diagnosis of all stages of gastric carcinoma with specificity ranged between (95\%-100\%) and accuracy ranged between (94.5\%-97.5\%). However, it showed that CT with MPR was specific and accurate in diagnosis of all stages of gastric carcinoma. However, it showed lowest sensitivity (75\%) in the diagnosis of stage I of gastric cancer. On the other hand it showed highest sensitivity (97.5\%) in diagnosis of stage 4.

\section{CASE (1)}

A 71-years old male patient presented with abdominal pain, vomiting and progressive loss of weight for 5 months.

- MDCT Findings: Post contrast portal phase axial (a , b , c), sagittal (d) and coronal (e) CT scans of the stomach revealed circumferential wall thickening of the gastroesophageal junction extended into the cardia of the stomach reaching about $3.5 \mathrm{~cm}$ associated with irregular focal gastric wall thickening seen at the fundus and lesser curvature, with no associated lymphadenopathy or extra-gastric spread.

- MDCT Diagnosis: Gastric carcinoma stage I.

- Histopathological Diagnosis: High grade invasive adenocarcinoma grade II-III
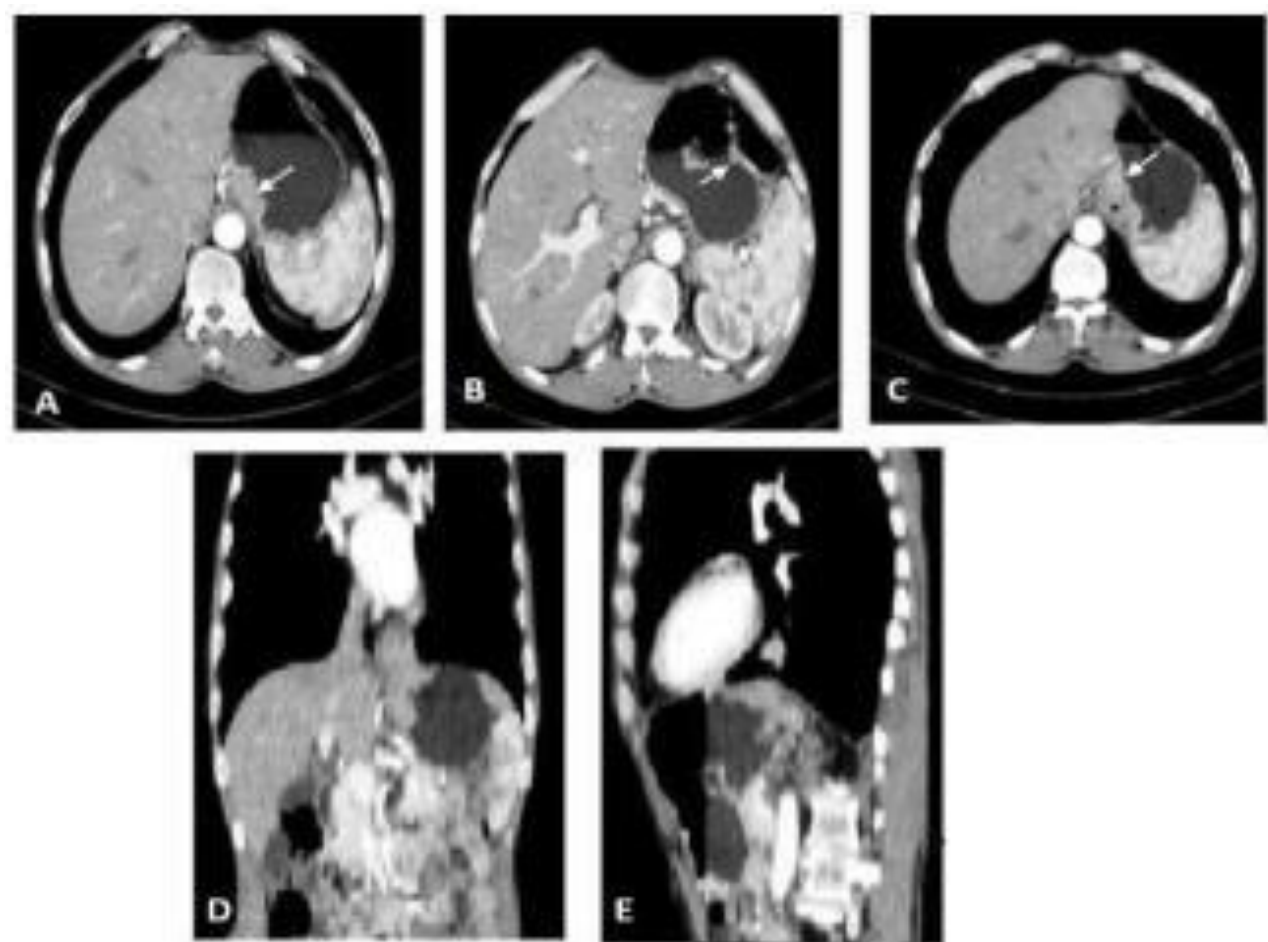

Figure (1): A, B, C) Axial views of the stomach at different levels D) sagittal reformatted image E) Coronal reformatted image 
CASE (2)

A 43-years-old female patient presented with vomiting and progressive weight loss for 4 months

- MDCT Findings: MDCT Findings: Post contrast axial (a and b), sagittal (c) and coronal (d) CT scans of the abdomen and pelvis revealed polypoidal focal thickening of the greater curvature of the stomach measured about $(4.5 \times 2.5 \mathrm{~cm})$ in its maximal dimensions with multiple enlarged paragastric and para-aortic LNs. No evidence of extragastric spread or distant metastasis could be noted suggesting gastric carcinoma stage III.

- Histopathological Diagnosis: Signet ring adenocarcinoma grade II to III
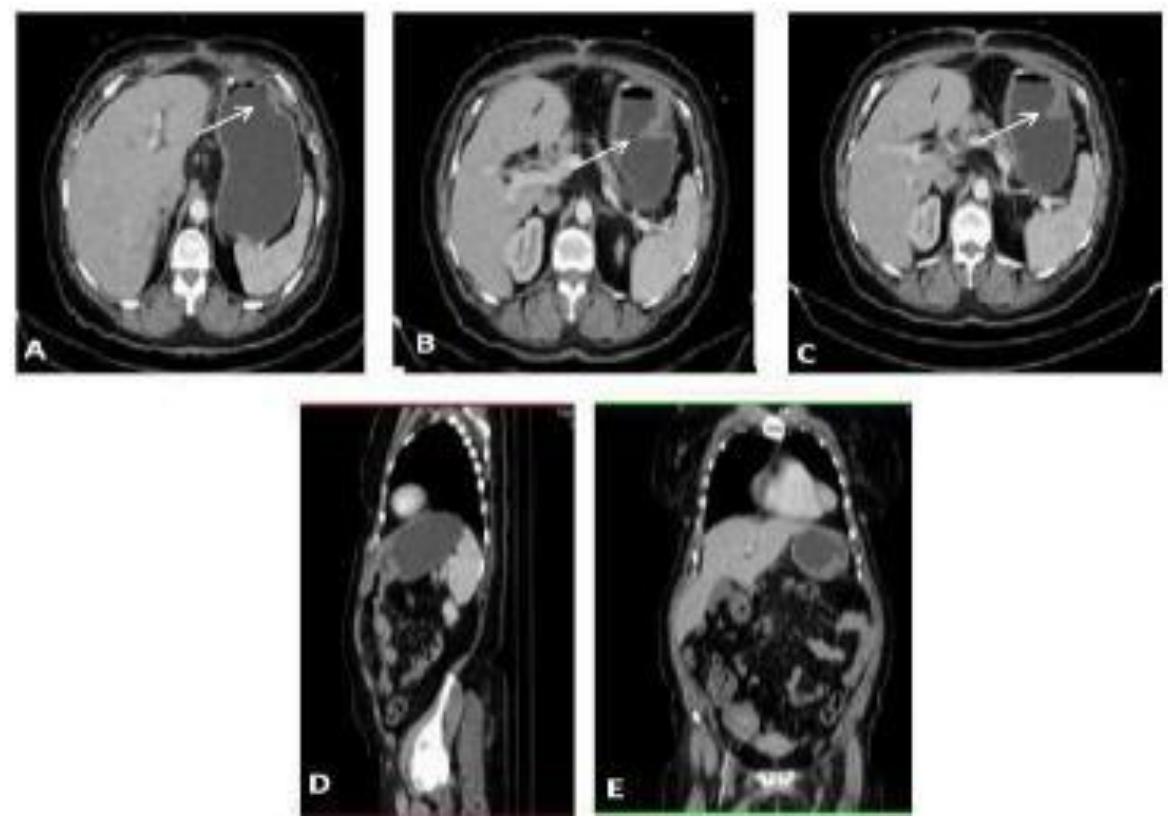

Figure (2): A, B, C) Axial views of the stomach at different levels D) sagittal reformatted image E) Coronal reformatted image

\section{CASE (3)}

A 53-years-old female patient presented with vomiting, dysphagia and progressive weight loss for 4 months

- MDCT Findings: MDCT Findings: Post contrast axial ( $a, b$ and c), sagittal (d) and coronal (e) CT scans of the abdomen and pelvis revealed circumferential mural thickening of the gastroesophageal junction reaching about $2 \mathrm{~cm}$ with focal wall thickening of the gastric fundus and lesser curvature of the stomach (long arow) measured about $(1.5 \mathrm{~cm})$ in its maximal dimensions with smudging of fat planes around it. Multiple enlarged perigastric, gastrohepatic, celiac and para-aortic LNs. (short arrow). No evidence of extragastric spread or distant metastasis could be noted suggesting gastric carcinoma stage III.

- Histopathological Diagnosis: Poorly differentiated adenocarcinoma grade III
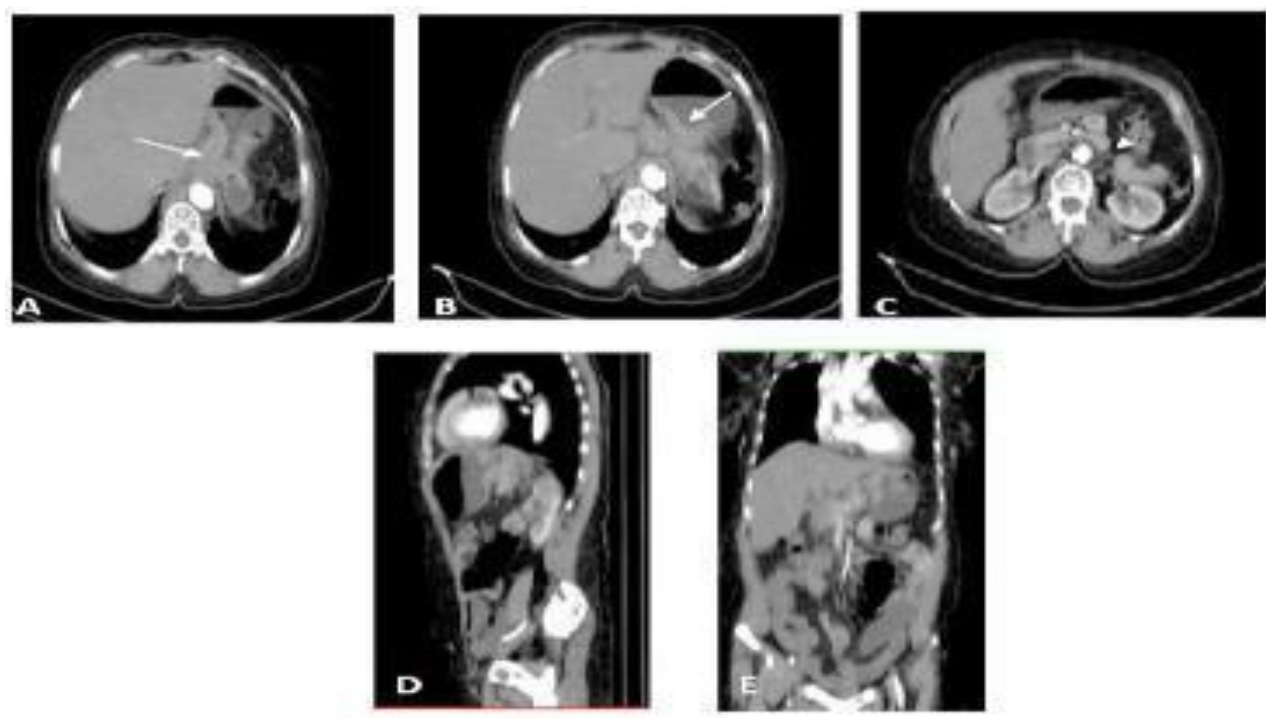

Figure (3): A, B, C) Axial views of the stomach at different levels D) sagittal reformatted image E) Coronal reformatted image 


\section{CASE (4)}

A 66-years-old female patient presented with marked weight loss and abdominal pain for 6months.

- MDCT Findings: MDCT Findings: Post contrast axial (a and b), sagittal (c) and coronal (d) CT scans of the abdomen and pelvis revealed diffuse irregular mural thickening of the stomach more at the fundus and body (large arrow) reaching about $3 \mathrm{~cm}$ in its maximal dimensions with loss of fat planes between the stomach and pancreas. With enlarged para-aortic LN (black arrow) suggesting gastric carcinoma stage III.

- Mild ascites

- Histopathological Diagnosis: Round cell malignancy grade IV
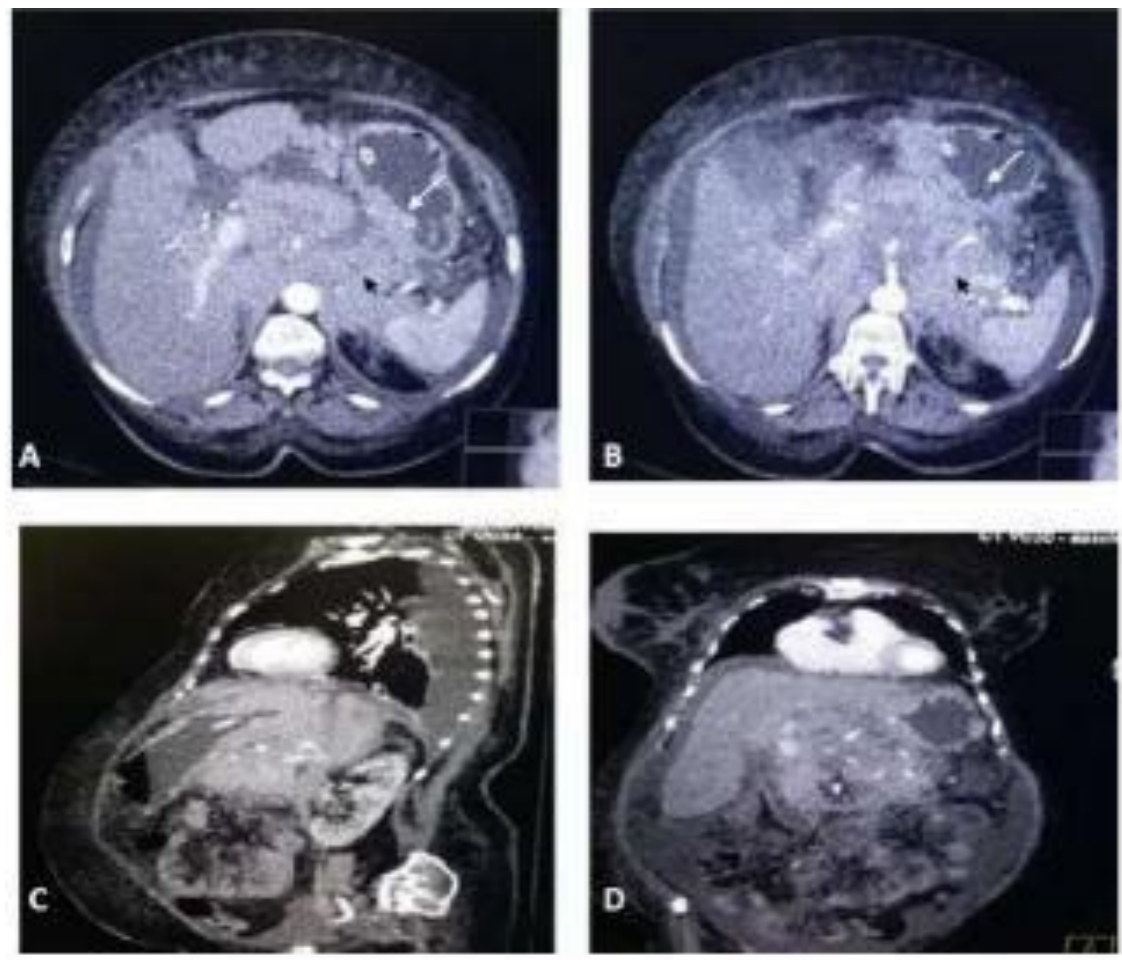

Figure (4): A, B) Axial views C) sagittal reformatted image D) Coronal reformatted image

\section{DISCUSSION}

The aim of this study was to clarify the role of multidetector computed tomography (MDCT) with water filling in diagnosis and evaluation of gastric cancer.

Although diagnostic advancements in performing endoscopy and double-contrast barium study currently allows the detection of small lesions early in the course of the disease, the depth of tumor invasion and the presence or absence of metastases could not be determined with either modality. In a routine clinical setting, patients with esophageal and gastric carcinoma most commonly undergo diagnosis and staging with computed tomography (CT) ${ }^{(4)}$.

MSCT scanner can be considered as a turbocharged spiral scanner. Conventional and spiral CT scanner use single-row detectors to pick up X-ray beam after it has passed through the patient. MSCT scanner has many rows of detectors and also comes with faster computer software, which offers high reconstruction and post processing capabilities. MSCT has allowed us to surpass most of the limitations of single-row detector spiral CT, in particular the very thin section collimation. The high speed of acquisition and the near isotropy of the voxels allow us to reformat images in any plane without substantial artifacts and with excellent anatomic details. A key advantage of MSCT is its speed. Combining of a multirow detector array and a reduced gantry time rotation allows scanning of a very large volume very quickly and therefore opens to CT many applications that were not possible with spiral $\mathrm{CT}^{(4)}$.

The use of water is essential in our study as there must be adequate distension of the stomach. If the entire stomach is not well distended, disease must be over looked or, conversely, the collapsed gastric wall may mimic disease. Traditionally, high attenuation contrast agent was administrated to enhance and distend the stomach and gastrointestinal tract, these agents can be categorized as positive contrast agent because they have CT attenuation greater than that of water. Although these agents are safe, well tolerated and result in good gastric distention, they may not be optimal when evaluating the gastrointestinal tract and the stomach. Sometimes positive oral contrast agents homogenously merge 
with gastric contents and pseudo-tumors can be obtained on axial image ${ }^{(6)}$.

The present study was conducted on 60 patients with age ranged from 28 to 73 years with mean age of 53 years. By analyzing the age distribution among patients with gastric carcinoma, we found that the ages of the patients diagnosed as having gastric carcinoma ranged from 28 to 76 years. The most frequently involved age group was the group > 50-70 years. This is in agreement with Teama et al. ${ }^{(4)}$ who found that stomach cancer mostly affects older people and the sixth decade group was the most affected age group. This is also agreed with the American Cancer Society ${ }^{(7)}$ who stated that there is a sharp increase in stomach cancer rates in people over age 50 .

In the present study we found that females were more affected (34 females) than males (26 male). This is in disagreement with Bray et al. ${ }^{(\mathbf{8})}$ who found that gastric cancer rates are 2-fold higher in men than in women. This is may be due to the geographic distribution.

In the current study, we found that the poorly differentiated adenocarcinoma is the most common gastric cancer that was diagnosed in 20 patients ( 33.3 $\%)$ followed by high grade invasive adenocarcinoma that was diagnosed in 15 patients $(25 \%)$, then both round cell and signet ring carcinoma that was diagnosed in 10 patients each $(16.7 \%)$. The least common was moderately differentiated adenocarcinoma that was diagnosed in 5 patients $(8.3 \%)$. This is in agreement with Liu et al. ${ }^{(\mathbf{9})}$ who observed that poorly differentiated adenocarcinoma was the most common type among the patients of their study, and also Shimizu et al. ${ }^{(10)}$ observed that the undifferentiated adenocarcinoma was the most common type among the patient of their studies.

In the CT study, the assessment of the gastric wall thickness is an integral part. Optimal distension of the stomach results in effacement of the normal folds. The normal gastric wall is thin usually measuring 5-7 $\mathrm{mm}$ when the stomach is well distended, wall thickness greater than $8-10 \mathrm{~mm}$ is definitely abnormal, and however, the wall of the fundus and antrum may appear thicker than the remainder of the stomach, because of their orientation within the scanning plane. The wall thickness of the cardia may appear thicker due to the fact that axial slices may intersect the curved gastric wall, measuring up to $12-15 \mathrm{~mm}^{\left({ }^{(11)} \text {. }\right.}$

In the current study, all sixty patients were scanned by multislice CT. Gastric mass appears in 21 cases $(35 \%)$ that included 11 male $(18.3 \%)$ and 10 females $(16.7 \%)$. The masses were polypoidal and fungating. Circumferential masses encircling the gastric wall and encroaching into the lumen were identified in $20(33.3 \%)$ patients (5 males and 15 females). Malignant focal wall thickening was detected in $15(25 \%)$ patients that included 8 males $(13.3 \%)$ and 7 female (11.7\%). This is in disagreement with Teama et al. ${ }^{(4)}$ who found that circumferential wall thickening of the stomach is the most common CT appearance of gastric cancers.

In the current study, we found that the most commonly affected site by gastric carcinoma was the cardia and gastroesophageal junctionwhich was found in $20(33.3 \%)$ patients. This is in agreement with Teama $e t$ al. ${ }^{(4)}$. This is also agreed with Liu $\boldsymbol{e t}$ al. ${ }^{(9)}$ who found that the cardia was the most common affected site with gastric cancer in their study.

In our study, the presence of distant metastasis was found in 32 patients $(53.3 \%)$ at the time of diagnosis and the liver was the mostly affected organ of distant metastasis that was found in 12 patients out of 32 patients with distant metastasis. This is agreed with Sun et al. ${ }^{(\mathbf{1 2})}$ who found that 35\% of patients presented with evidence of distant metastases at the time of diagnosis and about half of them had metastatic disease to the liver, the most common metastatic organ.

In our study we found that there is a significant relationship between pathological and CT staging by using thin axial CT as we found that $\mathrm{CT}$ was specific and accurate in diagnosis of all stages of gastric cancer with specificity ranged between (9397\%) and accuracy ranged between (90-92.5\%). However, it showed lowest sensitivity (50\%) in diagnosis of stage 1 of gastric cancer. On the other hand, it showed highest sensitivity (90\%) in diagnosis of stage IV. This is in agreement with Teama et al. ${ }^{(4)}$ who reported that CT has higher sensitivity in depiction and accurate staging of $\mathrm{T} 4$ $(85.7 \%)$ and $\mathrm{T} 3(83.3 \%)$ in comparison with $\mathrm{T} 2$ (55.6\%) and $\mathrm{T} 1(50 \%)$ staging. This is also in agreement with Sohn et al. ${ }^{(13)}$ who reported that MDCT has sensitivities in the range of $68.8 \%-96.2 \%$ in detection of gastric cancers, most of patients presented in grade IV of gastric cancers.

In our study we found that there is a significant relationship between pathological and CT staging by using MPR as we found that CT with MPR was specific and accurate in diagnosis of all stages of gastric cancer with specificity ranged between (95$100 \%)$ and accuracy ranged between (94.5-97.5\%). However, it showed lowest sensitivity in diagnosis of stage 1 of gastric cancer. On the other hand, it showed highest sensitivity (97.5\%) in diagnosis of stage IV. This is in agreement with Hallinan and Venkatesh ${ }^{(14)}$ who reported that with the advent of MDCT and the possibility of MPRs, the accuracy of evaluation of invasion of the gastric wall was significantly improved from $69-84 \%$ for single detector CT to 80 $89 \%$.

In our study we found that stage IV of cancer stomach was better recognized by using MPR sequences, while grade II and III of cancer stomach 
were better identified by axial cuts with thin slice MDCT. However, the difference between two sequences was not significant. These results are in agreement with Shimizu et al. ${ }^{(10)}$ who stated that MPR has superiority in detection of gastric cancers in different stages.

MSCT is currently the staging modality of choice, because it can help identify the primary tumor, assess for focal spread and the depth of tumor invasion, and detect nodal involvement and distant metastases ${ }^{(4)}$.

\section{CONCLUSION}

1. MDCT can be the first choice for the preoperative evaluation of patients with gastric carcinoma, with further imaging approaches considered in selected cases.

2. Detailed CT examination of the stomach can routinely be performed when water is used as an oral contrast agent, along with rapid intravenous contrast bolus and the thin collimation that is possible with new multidetector row CT scanners.

3. MSDT is a valuable tool for detection, staging, surveillance, and post-treatment evaluation of gastric neoplasm.

4. Recent advances in CT technology imaging software have sparked renewed interest in using CT to evaluate gastric neoplasm.

\section{REFERENCES}

1. Habermann CR, Weiss F, Riecken $R$ et al. (2004): Preoperative staging of gastric adenocarcinoma:comparison of helical CT and endoscopic US Radiology, 230:465-471.

2. Moschetta M, Stabile Ianora AA, Anglani A et al. (2010): Preoperative $T$ staging of gastric carcinoma obtained by MDCT vessel probe reconstructions and correlations with histological findings. Eur Radiol., 20:138-45.

3. Kwee RM and Kwee TC (2009): Imaging in assessing lymph node status in gastric cancer. Gastric Cancer, 12:6-22.

4. Teama AH, El-Badry AM, Yousef ES (2016): The role of multislice computed tomography in the diagnosis of gastric malignant tumors. Tanta Med J., 44:119-26.

5. Dixon M, Cardoso R, Tinmouth J et al. (2014): What studies are appropriate and necessary for staging gastric adenocarcinoma? Results of an international RAND/UCLA expert panel. Gastric Cancer, 17:37782.

6. Karen MH and Elliot KF (2003): Current Role of CT in Imaging of the Stomach. https://pubs.rsna.org/doi/full/10.1148/rg.231025071

7. American Cancer Society (2019): Key statistics about stomach cancer. American CancerSociety.Available at http://www. cancer.org/cancer/ stomach cancer/ detailedguide/stomach-cancer-key-statistics..

8. Bray F, Ferlay J, Soerjomataram I et al. (2018): Global Cancer Statistics 2018: GLOBOCAN Estimates of Incidence and Mortality Worldwide for 36 Cancers in 185 Countries. CA: Cancer J Clin., 68:394-424.

9. Liu S, Liu S, Ji C et al. (2017): Application of CT texture analysis in predicting histopathological characteristics of gastric cancers. Eur Radiol., 27(12): 4951-4959.

10. Shimizu $K$, Ito $K$, Matsubaga $N$ et al. (2010): Diagnosis of Gastric Cancer with MDCT using the water filling method and multiplanar reconstruction: CT- Histologic correlation. AJR., 185: 1152-1158.

11. Bean M, Horton $K$ and Fishman $E$ (2005): Detection and diagnosis of gastric carcinoma with multidetector and 3D computed tomography. Applied Radiology, 242:20- 30 .

12. Sun Z, Zheng H, Yu J et al. (2019): Liver Metastases in Newly Diagnosed Gastric Cancer: A PopulationBased Study from SEER. J Cancer, 10(13):2991-3005.

13. Sohn KM, Lee JM, Lee SY et al. (2010): Comparing MR imaging and CT in the staging of gastric carcinoma. AJR., 174:1551-1557.

14. Hallinan JT, Venkatesh SK (2013): Gastric carcinoma: imaging diagnosis, staging and assessment of treatment response. Cancer Imaging, 13(2):212-227. 